\title{
A Bayesian Approach to Dynamic Contours through Stochastic Sampling and Simulated Annealing
}

\author{
Geir Storvik ${ }^{1}$ \\ Mathematical Institute \\ University of Oslo \\ P.O.Box 1053, Blindern \\ N-0314 Oslo 3, Norway \\ email: geirs.@math.uio.no \\ Submitted for publication as a regular paper in IEEE PAMI
}

${ }^{1}$ Supported by Royal Norwegian Council for Scientific and Industrial Research, Grant ST.10.12.220289. 


\begin{abstract}
In many applications of image analysis, simply connected objects are to be located in noisy images. During the last 5-6 years active contour models have become popular for finding the contours of such objects. Connected to these models are iterative algorithms for finding the minimizing energy curves making the curves behave dynamically through the iterations. This approach do however have several disadvantages. The numerical algorithms that are in use constraint the models that can be used. Furthermore, in many cases only local minima can be achieved.

In this paper we discuss a method for curve detection based on a full Bayesian approach. Furthermore, iterative algorithms based on stochastic sampling makes it possible to simulate samples from the posterior distribution, making estimates and uncertainty measures of specific quantities available. Further, simulated annealing schemes making the curve move dynamically towards the global minimum energy configuration are presented. In theory, no restrictions on the models are made. In practice, however, computational aspects must be taken into consideration when choosing the models. Much more general models than the one used for active contours may however be applied.

The approach is applied to ultrasound images of the left ventricle and Magnetic Resonance images of the human brain, and show promising results.
\end{abstract}




\section{Introduction}

In most problems of image analysis, incorporation of prior knowledge is important for making inference based on the images. In the recent years there has been an increasing interest in use of statistical techniques for modeling and processing image data. In the Bayesian approach of statistical inference, the goal is to make proper use of the prior information that is available.

Images containing simply connected objects appear in many applications. Two examples that will be considered in this paper are ultrasound images of the left ventricle, where the left ventricle is a simply connected object and Magnetic Resonance images of the brain, where many different structures are simply connected objects. In both examples, recognizing the contours of these objects is of interest.

The traditional approach is to detect edges and then link them (Dufresne and Dhawan [7], Venkateswar and Chellappa [23], Tan et al. [22]). The knowledge that the object is simply connected is however not utilized in such approaches. Incorporation of such information by searching directly for the contours of such objects will be more natural. For such cases, "the need arises to determine a contour having optimal properties amongst a large space of functions" (Amini et al. [2]). Finding the optimal contour amounts to minimizing an objective function.

Active contours (Kass et al.[13], Amini et al.[1, 2]) have recently been developed for finding optimal contours in a neighborhood of an initial guess of the solution. In some cases, this neighborhood is sufficiently large so that the resulting solution will be the optimal one. In other cases, only a very small neighborhood is used, and the final contour is found through an iterative algorithm using the solution from the previous iteration as a starting-point. In this case, only local optimal solution will be found and dependence of the initial guess is then an unappealing feature. Furthermore, the numerical techniques at each iteration restrict the set of possible models that can be used.

In this paper a new method for contour detection is presented. Bayesian statistical theory makes a convenient way of taking a priori information into consideration when inference is to made from images (see Besag [3]). We will follow the Bayesian approach for developing models for simply connected objects. Furthermore, algorithms based on the Metropolis algorithm (Metropolis et al. [17], Ripley [19]) for simulation and simulated annealing (Geman and Geman [10]) for optimization will be presented. The use of such general techniques for performing inference, makes it possible to apply much more general models than the ones usually chosen for the active contour approach. In particular more global prior models for the shape of the simply connected object and models utilizing more of the available information in the observed image can be applied. Following the Bayesian approach, quantities can be estimated and uncertainty measures can be specified.

In Section 2, the use of vector-representation for contour detection and the active contour approach is discussed. Section 3 presents the new method for contour detection. Several examples of prior and data models are discussed, and algorithms for simulation and optimizing the posterior distribution are presented. In Section 4, the approach is applied to ultrasound images of left ventricle and MR-images of the brain. Summary and discussion are provided in Section 5 . Proofs of convergence of the algorithms presented in section 3 appear in the appendix.

\section{Background}

We describe the current approaches for detecting the contours of simply connected objects in this section.

Under the assumption that the image consists of only one object having a simply connected domain, the image $x$ of interest is completely defined by the contour of the object. A polygon (or vector) representation of an object is a representation where the contour is defined by a set of nodes giving coordinates of points on the contour in a circular (clockwise) manner. Between each node, the contour is defined by a straight line (or perhaps some spline-curves). Figure 1 illustrates the representation. 


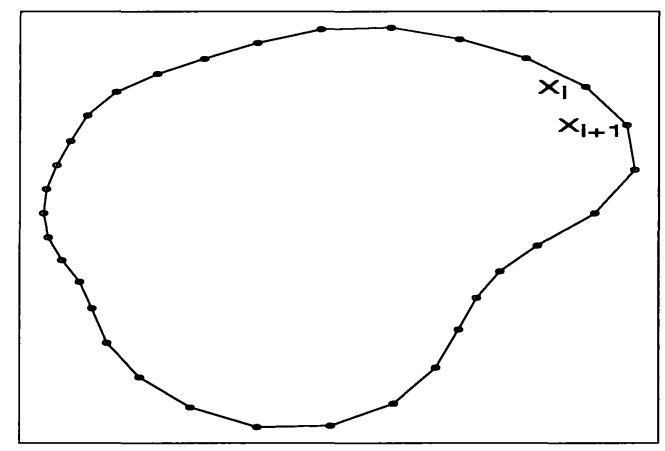

Figure 1: Polygon-representation of the contour for a simply connected object.

Representation of objects in this manner has been widely considered in the object recognition literature (Persoon and Fu [18], Zahn and Roskies [25]). The benefit of using such representations in the segmentation step of image analysis has been recognized in the active contour approach (Kass et al. [13], Amini et al.[1, 2]) and also for the "radii-based" approach (Friedland and Adams [8], Lundervold and Taxt [16]). Representing the image by contour nodes rather than image pixels incorporates the postulated simple connectivity, and drastically reduces the dimension of the configuration space. For both the active contour and the radii-based approaches, a fixed number of nodes are used in the contour representation, and the contour is then defined through the locations of the nodes. For the "radii-based" approach, a node on the contour is restricted to have possible locations only on a radii from a pre-specified centerpoint. Other types of restrictions are possible in order to simplify modeling and calculation.

The number of nodes used in the active contour approach is always fixed. This may in many cases be too restrictive when more complex structured contours are to be recognized. For some implementations of the active contour approach, the number of nodes can be random. Usually this is however done in rather ad hoc ways.

Prior knowledge is usually present on the contours to be recognized (note that prior information on the object being simply connected is already built in to the representation of the true image). The Bayesian approach is a formal theory for incorporation of such information. Use of this approach in image analysis has been quite popular in the recent years. Besag [3] defines the Bayesian paradigm to consist of four successive stages:

1. Construction of a prior probability distribution $\pi(x)$ where $x$ is to be reconstructed (in our case the contour of the object).

2. Combining the observed image $z$ with the underlying contour $x$ through a conditional probability density $f(z \mid x)$.

3. Constructing the posterior density $p(x \mid z)$ from $\pi(x)$ and $f(z \mid x)$ by Bayes Theorem giving

$$
p(x \mid z) \propto \pi(x) f(z \mid x) .
$$

4. Base any inferences about $x$ on the posterior distribution $p(x \mid z)$.

In Bayesian analysis, all kind of inference is made from $p(x \mid z)$. Finding the maximum a posteriori (MAP) estimate is one choice of inference. Other quantities may however also be important. Estimates of certain quantities (such as volumes) can be of interest, and usually uncertainty measures of these estimates are required. 
For the active contour approach, the need for incorporation of smoothness constraints on the contours is recognized. Constraints on contours are for this approach designed through energyfunctions. The energy-function consists of internal and external forces, where the internal forces serve as a smoothness constraint and the external forces guide the active contour towards image features (Kass et al. [13]). Although rarely mentioned, the use of such energy-functions fall into the Bayesian framework. Assume $U(x)$ is the total energy of the contour represented by $x$, and assume

$$
U(x ; z)=U_{i n t}(x)+U_{e x t}(x ; z)
$$

where $U_{\text {int }}$ is the internal force while $U_{\text {ext }}$ is the external force (depending on the observed image $z$ ). Define then a prior model for $x$ by

$$
\pi(x)=\frac{1}{Z_{i n t}} e^{-U_{i n t}(x)}
$$

where $Z_{\text {int }}$ is a normalization constant guaranteeing the prior to be a proper probability distribution. Assume further that the likelihood for the observed image $z$ given $x$ is defined by

$$
f(y \mid x)=\frac{1}{Z_{e x t}} e^{-U_{e x t}(x ; z)}
$$

where $Z_{\text {ext }}$ is a normalization constant similar to $Z_{\text {int }}$. The posterior probability for the contour conditioned on the image is then

$$
\pi(x \mid z) \propto \pi(x) f(z \mid x) \propto e^{-U_{i n t}(x)-U_{e x t}(x ; z)}=e^{-U(x ; z)}
$$

showing that minimizing the energy function corresponds to maximizing the posterior distribution, that is finding the Maximum a posteriori (MAP) solution.

Optimal contours are usually impossible to find directly. Therefore, iterative algorithm that dynamically moves the contour towards the optimal solution has been proposed. For the active contour approach (Kass et al. [13], Amini et al. [1, 2]), an initial contour is defined through specifying the location of $N$ nodes. Various algorithms based on dynamic programming or variational methods are constructed for finding the optimal (minimum energy) contour in a neighborhood of the initial contour. In some cases, this neighborhood is large enough so that the given solution will be the desired one. In many situations, however, only a very small neighborhood is used, and the final contour is found through an iterative algorithm using the solution from the previous iteration as a starting-point. In such a case, only a local optimal solution will be found and dependence of the initial guess is an unappealing feature.

Another drawback with these algorithms is that the numerical techniques used at each iteration restrict the set of possible models (or equivalently, energy-functions) that can be applied. In particular, the external forces in all applications known to the author are using only gradient (or some other local) information along the contour. This makes it difficult to know which direction the contour should move when it is inside a homogeneous region. Some attempts have been made for solving this problem. Leitner et al. [14] and Cohen et al. [5] discuss the use of distance measure to nearest edge-points for speeding up the convergence.

\section{A new method for Contour detection}

In this section we describe a new method for contour detection. The approach differs from the other approaches in three ways:

- The number of nodes used for representing the contour is random,

- more flexible models both for the prior and for the likelihood are applied, 
- new algorithms for simulation and optimization from the posterior distribution are constructed.

We will show how algorithms based on stochastic sampling may be constructed both for simulation from and maximization of $p(x \mid z)$. The simulation algorithms can be used to estimate quantities through Monte Carlo methods, while maximization algorithms can be used for finding the MAP estimate. This will be discussed in section 3.3. We will however start discussing possible prior models. Models for the observed image $z$ will be discussed in section 3.2.

\subsection{Prior models}

Consider now models for the prior distribution $\pi(x)$. Energy-functions are usually easier to formalize than probabilities. We therefore assume that $\pi(x)$ is of the form

$$
\pi(x)=\frac{1}{Z_{\alpha}} e^{-\alpha^{\prime} U(x)},
$$

where $\alpha^{\prime} U(\cdot)$ is called the energy function. The vector $U(\cdot)=\left\{U_{1}(\cdot), \ldots, U_{p}(\cdot)\right\}$ contains components measuring various characteristics of the contour, while $\alpha$ is a parameter vector containing the weights that are given on the different components. $Z_{\alpha}$ is a normalizing constant, usually unknown because of the huge number of possible configurations $x$.

The a priori distribution should capture the knowledge available about $x$. A common assumption is that the energy-function is built up by potentials measuring local characteristics. In that case,

$$
U(x)=\sum_{i} V_{i}(x)
$$

where the sum is over all nodes on the contour and $V_{i}(x)$ is some measure depending only on the nodes in a small neighborhood of node $i$. In the case of random number of nodes, an alternative could be to use the average of the potential measures,

$$
U(x)=\frac{1}{|x|} \sum_{i} V_{i}(x)
$$

where $|x|$ is the number of nodes on the contour $x$. As noted above, the total energy consists of many (additive) energy-functions. In the following we will discuss several choices of such functions. Each function can be used alone or in combination with other functions. For simplicity we suppress the subscript of the energy-functions.

Smoothness is the most common aspect used to incorporate in this distribution. For the active contour approaches, the derivatives and second derivatives of the contour have been used as smoothing measures. The derivatives are approximated by

$$
\begin{aligned}
V_{i}^{1}(x) & =\left|x_{i}-x_{i-1}\right|^{2}, \\
V_{i}^{2}(x) & =\left|x_{i+1}-2 x_{i}+x_{i-1}\right|^{2} .
\end{aligned}
$$

which are used as potentials in the energy function. As noted by Williams and Shah [24], minimization of the energy corresponding to the first derivative has the effect of causing the contour to shrink, since the energy will be a discrete version of the contour length.

Also the discrete version of the second derivative will be scale-dependent, but this dependence may be removed by dividing the potential by the distance between node $i$ and node $i+1$.

An alternative potential function, measuring the curvature of the contour, was proposed by Williams and Shah [24]:

$$
V_{i}(x)=\cos ^{-1} \frac{\left(x_{i}-x_{i-1}\right) \cdot\left(x_{i+1}-x_{i}\right)}{\left|x_{i}-x_{i-1}\right|\left|x_{i+1}-x_{i}\right|}
$$


that is the angle between the two vectors $x_{i}-x_{i-1}$ and $x_{i+1}-x_{i}$. A similar measure was given in Storvik and Switzer [21]. Calculations performed on examples showed this potential function to perform quite well (Williams and Shah [24]). In Storvik and Switzer [21] several other possible potential functions were proposed.

In some cases, energy-functions that can not be split into potentials may be of interest. One such is the following:

$$
U(x)=\frac{(\text { contour length })^{2}}{\text { area of object }} .
$$

This measure is sometimes called the fractal dimension of the object.

\subsection{Probability densities for data}

The probability density $f(z \mid x)$ is related to the specification of the observed image data. In Kass et al. [13] and Amini et al. [1, 2], energy-functions connecting the contour to the image features were defined through potentials along the contour, that is

$$
E(x, z)=-\sum_{i} h\left(x_{i} ; z\right)
$$

where $h\left(x_{i} ; z\right)$ is some local measure from the observed image $z$ at location $x_{i}$. Examples are the grey-levels itself or some gradient measure. The use of such energy-functions corresponds to assuming a likelihood for $z$ given $x$ by

$$
f(z \mid x)=\frac{1}{Z} \prod_{i} e^{-h\left(x_{i} ; z\right)} .
$$

That is, measurements only in the neighborhood of the contour $x$ are influenced by $x$. This assumption leads to a large simplification in the calculations and is crucial for algorithms based on dynamic programming to work.

In many cases, not only the observed measurements along the contour but also the greylevels inside and outside the object contain information, for instance when the average greylevel inside the object is very different from the average grey-level outside the object. Neglecting such information may lead to sub-optimal solutions in addition to slower convergence for the algorithms. In particular, when only gradient information is used, an inital contour which is not close enough to the image features is not attracted by them (Cohen [6]), making problems for a dynamic algorithm to know which directions to move the contour when searching for the optimal solution.

An alternative model is to assume one distribution function $f_{1}$ for the pixels inside the object, and another distribution function $f_{2}$ for the pixels outside the object. In that case,

$$
f(z \mid x)=\prod_{j \in R_{1}} f_{1}\left(z_{j}\right) \prod_{j \in R_{2}} f_{2}\left(z_{j}\right)
$$

where $R_{1}$ and $R_{2}$ are the set of pixels inside and outside the contour, respectively, where $R_{1}$ and $R_{2}$ are specified by $x$. Note that for such models, extension to multispectral images $z$ is straightforward. Particular choices for $f_{i}(x)$ could be the Gaussian distribution

$$
f_{i}=N\left(\mu_{i}, \Sigma_{i}\right)
$$

or, more generally, the mixture Gaussian distribution

$$
f_{i}=\sum_{k=1}^{K_{i}} \pi_{i, k} N\left(\mu_{i, k}, \Sigma_{i, k}\right)
$$

where $\sum_{k=1}^{K_{i}} \pi_{i, k}=1$. 


\subsection{Algorithms for simulation and MAP estimation}

We will in this section construct algorithms for simulation and optimization for the models involved. For simplicity, we assume the space of possible contours is finite (one such restriction is considered in section 3.4). An extension to more general configuration spaces is straightforward. For simplification in notation, we assume the model is given by

$$
\pi(x)=\frac{1}{Z} e^{\{-U(x) / T\}}
$$

where $Z$ is a normalization constant ensuring the probability-function to be a proper distribution function. The constant $T$ is usually referred to as the "temperature". The probability distribution $\pi(x)$ may in some cases be a prior distribution as discussed in section 3.1 in which $U(x) / T$ in (17) corresponds to $\alpha^{\prime} U(x)$ in (5), but more often a posterior distribution $p(x \mid z)$ where $U(x) / T$ in (17) corresponds to $\alpha^{\prime} U(x)+\beta^{\prime} E(x, z)$. Define

$$
\Omega=\{x ; x \text { is a contour with simply connected domain }\} .
$$

We assume that $\pi(x)>0$ for all $x \in \Omega$. Because of the large number of possible configurations, $Z$ is typically unknown. This makes direct simulation or optimization difficult. The reconstruction methods corresponding to active contours solve this by defining an initial guess on the contour $x$ and then searching for a local minimum in a neighborhood of the initial guess using either variational methods or dynamic programming.

The use of sampling techniques for simulation and optimization in image analysis problems was introduced in Geman and Geman [10]. Direct sampling is usually not possible due to the complex nature of the models involved, however iterative algorithms such as the Metropolis algorithm (Metropolis et al. [17], Ripley [19]) and the Gibbs sampler (Geman and Geman [10]) have been widely considered in the image analysis literature. These algorithms are based on constructing Markov Chains with state space $\Omega$ that are easy to simulate from and which have $\pi(x)$ as limiting distribution. Furthermore, the MAP estimate can be obtained by simulated annealing (SA) letting the temperature $T$ decrease during the simulation (see [10]).

Both the Metropolis algorithm and the Gibbs sampler are iterative procedures depending on the ratio $\pi(y) / \pi(x)$ for a transition from state $x$ to state $y$ at each iteration step. This is crucial because the normalizing constant $Z$ in (17) then disappear. A further requirement is however that $x$ and $y$ should be almost similar (i.e. differ only locally) in order to make $U(x)-U(y)$ simple to calculate. Geman and Geman [10] considered transition schemes where $x$ and $y$ differed only at one pixel. Under the MRF models, $U(x)-U(y)$ depend only on $x$ and $y$ (and $z$ if $\pi(x)=p(x \mid z)$ ) in the neighborhood of the pixel that they differ, making calculation easy. Each pixel is visited in either a random or deterministic way for possible changes.

In our case, a more natural approach will be to construct an iterative scheme where the changes at each step is done locally on the contour $x$, that is a dynamic behavior for the contour. Such a procedure has been applied for the active contour approach, based on the calculus of variation [13] or dynamic programming [1, 2]. We will follow this idea, but use the approach of the Metropolis algorithm. As noted above, this algorithm is based on constructing an artificial Markov Chain that has the desired distribution as the limiting distribution. For simulation, this distribution would be the posterior distribution, while for optimization, the limiting distribution would be the uniform distribution over the set of global maxima of the posterior distributions. There are several advantages of using sampling based algorithms compared to the algorithms used for the active contour approaches:

- Weaker assumptions has to be made on the models that can be applied.

- Not only the optimum, but any quantity from the posterior distribution may be obtained through Monte Carlo sampling.

Construction of a Metropolis algorithm mainly involves the definition of a transition-matrix defining the possible transitions at each iteration step (see Ripley [19]). The transition matrix 
has to be constructed such that the resulting Markov chain $\{X(s)\}$ will be irreducible and aperiodic. Usually, because of implementational issues, changes from one iteration to another are local, that is only a small part of the configuration is changed. We follow this convention, by considering only changes for a small part of the contour at each transition.

A transition from one configuration to another can be defined through two steps, one defining the position at which the change is to be made, and another defining the type of change. When the number of sites (nodes in our case) is fixed, construction of algorithms where only one site is changed at a time, is easy to construct. Hastings [12] considered the case when co-ordinates (sites) are visited in a fixed sequence, while Geman and Geman [10] considered more general visiting scheme.

Randomness in the number of sites complicates the matter. In particular, making changes on $x$ by moving only a node from one location to another will not suffice. A technique for simplifying this problem is however to extend the configuration space $\Omega$ by introducing a stochastic variable indicating the position of a (fictional) object moving around the curve. That is, we are defining the extended configuration space

$$
\Omega^{*}=\left\{x^{*}=\left(x, p^{x}\right) ; x \in \Omega, p^{x} \in x\right\}
$$

where $p^{x}$ is the position node and $p^{x} \in x$ means that $p^{x}$ is a node on the contour $x$.

Conditioned on a configuration, the position node is assumed to have a uniform distribution on the set of nodes. The distribution is then given by

$$
\pi^{*}\left(x^{*}\right)=\frac{1}{Z} e^{-U(x) / T} \frac{1}{|x|}
$$

where $|x|$ is the number of nodes in configuration $x$. Note that the marginal distribution of $x$ is identical to the distribution given in (17) which means that a simulation of $\left(x, p^{x}\right)$ from $\pi^{*}(\cdot)$ produces a sample $x$ from $\pi(\cdot)$.

The changes possible at each iteration step are specified by the change matrix $Q^{*}$ where now $Q^{*}$ is defined over the extended configuration space $\Omega^{*}$. We assume that any transition is made through two steps:

1. A local change of the contour is made at the current position.

2. A change of position is made

Transitions of the first type are defined through the transition matrix $Q$, while transitions of the second type are defined through $R$. An algorithm for simulation from $\pi(x)$ is then the following:

Algorithm 1 Start with an arbitrary $X^{*}(0)$. For each iteration $s$ carry out the following steps:

1. Assume that $X^{*}(s)=x^{*}=\left(x, p^{x}\right)$ and select state $y^{*}=\left(y, p^{x}\right)$ by the distribution given by the $x^{*}$ th row of $Q$;

2. Change the contour to $z=y$ with probability $\alpha_{x^{*} y^{*}}$ while retain $z=x$ with probability $1-\alpha_{x^{*} y^{*}}$, where

$$
\alpha_{x^{*} y^{*}}=\frac{s_{x^{*} y^{*}}}{1+\frac{\pi^{*}\left(x^{*}\right)}{\pi^{*}\left(y^{*}\right)} \frac{q_{x^{*} y^{*}}}{q_{y^{*} x^{*}}}}
$$

and $S=\left\{s_{x^{*}}, s_{y^{*}}\right\}$ is a positive symmetric matrix.

3. Draw a new position node $p^{z}$ using the $\left(z, p^{x}\right)$ th row of $R$ and put $X^{*}(s+1)=\left(z, p^{z}\right)$.

A choice for $s_{x^{*}, y^{*}}$ of particular interest is

$$
s_{x^{*}, y^{*}}= \begin{cases}1+\frac{\pi^{*}\left(x^{*}\right) q_{x^{*} y^{*}}}{\pi^{*}\left(y^{*}\right)} & \text { if } \frac{\pi^{*}\left(y^{*}\right) q_{y^{*}} x^{*}}{\pi^{*} x^{*} x^{*} x^{*}} \geq 1 \\ 1+\frac{\pi^{*}\left(y^{*}\right) q_{y^{*}} x^{*}}{\pi^{*}\left(x^{*}\right) q_{x^{*}} y^{*}} & \text { if } \frac{\left.\pi^{*} * y^{*}\right) q_{y^{*} x^{*}}}{\pi^{*}\left(x^{*}\right) q_{x^{*} y^{*}}} \leq 1\end{cases}
$$


in which $\alpha_{x^{*}, y^{*}}$ reduces to

$$
\alpha_{x^{*}, y^{*}}=\min \left\{1, \frac{\pi^{*}\left(y^{*}\right) q_{y^{*} x^{*}}}{\pi^{*}\left(x^{*}\right) q_{x^{*} y^{*}}}\right\}
$$

Let $Q R$ denote the transition matrix defined by the pair $Q, R$. Criteria for convergence of this algorithm is stated in the following:

Theorem 1 Assume that $X^{*}$ is given by algorithm 1 and that the following conditions are satisfied:

1. The transition-matrix $Q R$ is irreducible and aperiodic.

2. $Q$ satisfies the similarity of position condition

$$
q_{x^{*} y^{*}}>0 \Rightarrow p^{x}=p^{y}
$$

3. $Q$ satisfies the symmetry of positivity condition

$$
q_{x^{*} y^{*}}>0 \Rightarrow q_{y^{*} x^{*}}>0 .
$$

4. $R$ is double stochastic, that is

$$
\sum_{x^{*}} r_{x^{*} y^{*}}=1
$$

Then the distribution of $X^{*}(s)$ simulated according to algorithm 1 converges to $\pi^{*}\left(x^{*}\right)$ when $s \rightarrow \infty$.

The proof of this Theorem is given in the appendix. Note that by not allowing the pathological case where $\alpha_{x^{*}, y^{*}}=1$ for all $x \neq y$, aperiodicity of $Q R$ follows if $R$ is aperiodic on each subset $\Delta_{x}=\left\{x^{*} ; x^{*}=\left(x, p^{x}\right), p^{x} \in x\right\}$.

The first assumption in Theorem 1 is the usual requirement of irreducibility and aperiodicity for convergence of Markov Chains. The second requirement is a consequence of separating the transition into two independent steps. The main idea is that the change matrix $Q$ makes changes only on the configuration and do not effect the position node. The assumption does however also make restrictions on the changes in that changes making the position node being removed from the contour are not allowed (which could be possible due to the randomness in the number of nodes).

The symmetry of positivity condition is necessary in order to calculate the probabilities $\alpha_{x^{*}, y^{*}}$. Note that in general, the total transition matrix $Q^{*}$ will not satisfy the symmetry of positivity condition. This is a generalization of the Hastings algorithm.

The assumption of $R$ being double stochastic is a somewhat stronger requirement than the requirement of each site being visited infinitely often in Geman and Geman [10], and could probably be relaxed.

A crucial part in the algorithm described above is the change matrix $Q$. Typically, the choice of $Q$ influences on the speed of convergence. Furthermore, some care has to be taken in order to satisfy the symmetry in positivity condition (25). For computational reasons, $x$ usually differs from $y$ only on a small part of the contour starting at node $p^{x}$. We will discuss some choices of $Q$ and $R$ in the next subsection.

Consider now maximization of $\pi(x)$. In this case, only the situation $\pi(x)=p(x \mid z)$ is natural to consider. Finding the MAP estimate by analytical methods is however prohibitive because of the huge computational cost. Geman and Geman [10] suggested the use of simulated annealing for constructing an iterative algorithm that converge to the solution. The method is again based on simulation of a Markov chain, but now constructing it so that the equilibrium is the 
MAP-solution. This is done by "cooling" down the "temperature" under the iterations. That is, instead of at each step $s$ using the model $\pi(x)$ given in (17), one uses

$$
\pi(x ; s) \propto e^{-U(x) / T(s)}
$$

where $T(s)$ is a decreasing sequence. The choice of $T(s)$ is a delicate one. Choosing a too fast decreasing sequence result in convergence to a local optima, while decreasing slow enough to obtain global maximum may be computationally prohibitive. For the Gibbs sampler approach, Geman and Geman [10] showed that a sufficient criterion for convergence is that $T(s) \geq c / \log (s+$ 1) provided $c$ is larger than the difference between the maximum and minimum of the energyfunction $U(x)$. Our models will not fit into the framework of Markov Random Fields and therefore the convergence results obtained in [10] will not cover our case. An algorithm with the similar convergence results is however possible to construct:

Algorithm 2 Start with an arbitrary $X^{*}(0)$. For each iteration $s$ carry out the following steps:

1. Assume that $X^{*}(s)=x^{*}=\left(x, p^{x}\right)$.

2. Select a state $y^{*}=\left(y, p^{x}\right)$ by the distribution given by the $x^{*}$ th row of $Q$.

3. Change the contour to $z=y$ with probability $\alpha_{x^{*} y^{*}}(s)$ and retain $z=x$ with probability $1-\alpha_{x^{*} y^{*}}(s)$, where

$$
\alpha_{x^{*} y^{*}}(s)=\min \left\{1, \frac{\pi(y ; s)}{\pi(x ; s)}\right\}
$$

and

$$
\pi(x ; s)=\frac{1}{Z_{s}} e^{-U(x) / T(s)}
$$

4. Draw a new position node $p^{z}$ using the $\left(z, p^{x}\right)$ th row of $R$ and put $X^{*}(s+1)=\left(z, p^{z}\right)$.

Note that in this case, the probabilities $\alpha_{x^{*}, y^{*}}$ are related to $\pi$ and not $\pi^{*}$. This is because optimization of $\pi$ is not equivalent to optimization of $\pi^{*}$. The convergence result for this algorithm is stated in the following Theorem:

Theorem 2 Assume $X^{*}(s)$ is given by algorithm 2 and that the following assumptions are satisfied:

1. The transition matrix $Q R$ is irreducible.

2. $Q$ satisfies the symmetry in positivity condition

$$
q_{x^{*} y^{*}}>0 \Rightarrow q_{y^{*} x^{*}}>0 .
$$

3. $Q$ satisfies the similarity in position condition

$$
q_{x^{*} y^{*}}>0 \Rightarrow p^{x}=p^{y} .
$$

4. Each sub-matrix of $R$ corresponding to a specific $x$ is an irreducible transition matrix.

5. The temperature sequence $\{T(s)\}$ satisfies

$$
T(s) \geq \frac{c}{\log (s+1)} .
$$

Let $\Omega^{M}$ denote the set of global minima of $U(x)$. Then, for $c$ large enough, 
1. for any state $x$ that is not a local minimum of $U(x), \lim _{s \rightarrow \infty} \operatorname{Pr}[X(s)=x]=0$.

2. $\lim _{s \rightarrow \infty} \operatorname{Pr}\left[X(s) \in \Omega^{M}\right]=1$.

The proof of this Theorem is deferred to the appendix. A major problem when applying simulated annealing is the slow convergence of the annealing schedule $\{T(s)\}$ and the specification of the constant $c$ (which usually will be very large). Cantoni [4] discusses the optimal schedules to be used when a finite number of iterations is performed (which in practice always will be the case). In that case, exponential schedules shows to be optimal.

\subsection{A specific choice of algorithm}

When implementing the approach discussed in the previous sections, care has to be taken in order to keep the computational cost reasonable. For a given model, the computational considerations is influenced by the choice of both contour representation and algorithms. In this section, we discuss one choice for vector representation and an algorithm which is efficient when using models of the type (14). This algorithm has been applied on the examples presented in section 4 .

To make the computation of models such as (14) easy, we assume that each pixel in the observed image $z$ will either be completely inside or completely outside the contour. Furthermore, the length between each node on the contour is assumed to be fixed and equal to the side-length of the pixels (assuming pixels to have quadratic shapes). This restriction forces the contour to follow the pixel-sides. Furthermore, the set of possible locations of nodes is restricted to the corners of the pixels. Figure 2 shows an example of such a configuration.

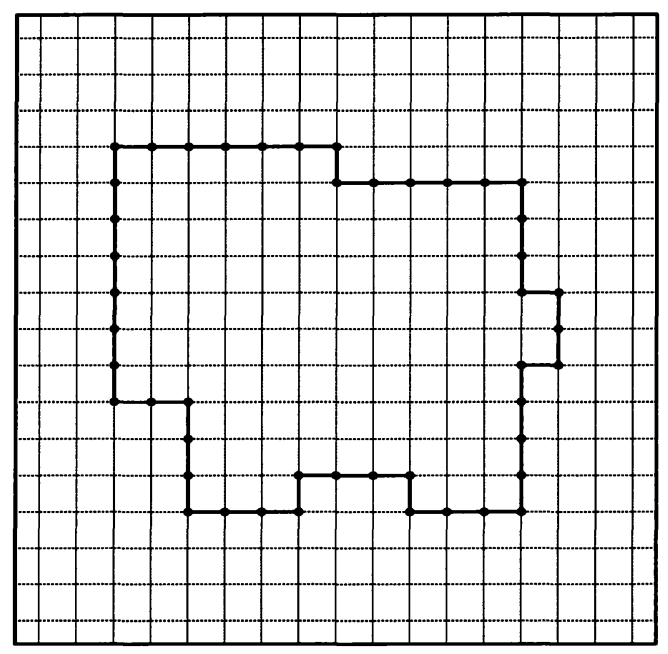

Figure 2: Example of a configuration when the contour follows the pixel-sides.

We will discuss some choices for possible changes from a state $x^{*}=\left(x, p^{x}\right)$. Assume $p_{x}=x_{p}$. The simplest approach is of course just the one where one pixel is changed at a time. In this case only two new configurations are possible, corresponding to the change of the two pixels having border on the line between the nodes $x_{p}$ and $x_{p+1}$. We will however not allow all such changes. Figure 3 shows two examples where problems occur if no restrictions are made. In the first example, a change of the pixel would lead to an illegal configuration; the object will no longer be simply connected. In the second case, the change of the pixel causes the position node to be excluded from the contour. We assume that neither of these cases are allowed. Note however that the restriction of not allowing any changed resulting in exclusion of the position node is no 
real restriction. Any such changes in the configuration can be performed from another position node.
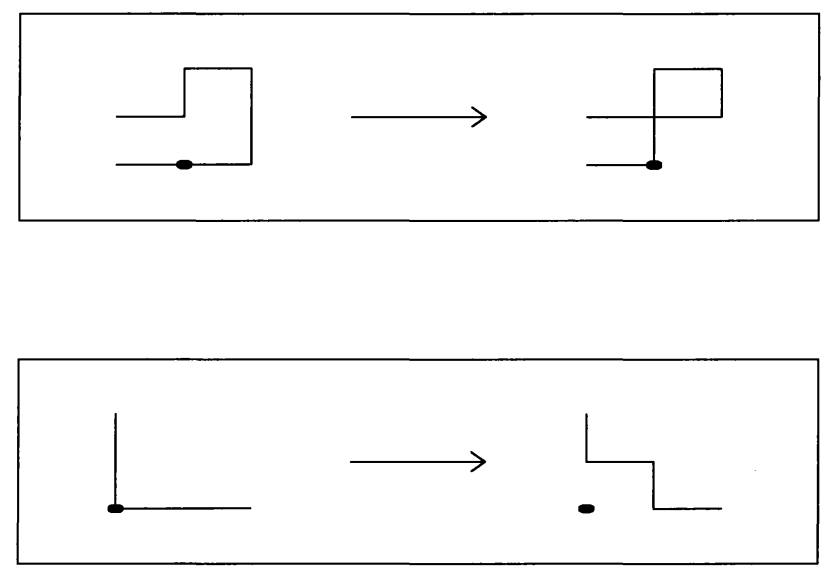

Figure 3: Example of changes causing problems.

Construction of algorithms allowing changes of more than one pixel at a time is more difficult. Gelfand and Smith [9] discussed the possibility of changing two pixels at a time, considering every possible pair of pixels in the image. We will instead consider algorithms that change parts of the contour at a time.

Consider first the case of two pixel-changes. We assume the two pixels to be changed are neighbors and that neither changes resulting illegal configurations nor changes that exclude the position node from the contour are allowed. Furthermore, the two pixels are assumed to be in the same region (both inside or both outside the contour) before a change is performed. Similar to the case of only one pixel-change, the first pixel is assumed to be adjacent to the line between the nodes $x_{p}$ and $x_{p+1}$. There are then three possibilities for the second pixel to be changed, as illustrated in the upper left plot of Figure 4. All these three possibilities have to be included in order to satisfy the symmetry of positivity condition (25) (if they result in legal transitions). For many configurations, some of the three possibilities will not result in a legal transition. Figure 4 shows some configurations and the legal transitions in each case.

Allowing transitions of three pixels at a time complicates matter. Again, the first pixel is assumed to be adjacent to the line between the nodes $x_{p}$ and $x_{p+1}$. In this case, 10 possibilities exists for the positions of the second and third pixels. In general, different number of pixels may be changed at each iteration. In order to fulfill the requirement of irreducibility, the greatest common divisor of the possible numbers has to be 1 .

For all the cases considered, the $Q$-matrix is defined by first choosing the number of pixels to be changed (from a fixed distribution $F(\cdot)$, usually the uniform distribution over the numbers of possible pixels to be changed). Then a draw is performed to determine whether the pixels should be moved from the inside to the outside of the contour or from the outside to the inside of the contour. Finally, a draw for the type of shape change has to be made. Such a specification is made by specifying the position (relative to the position node) of the pixels to be changed. A table of possible changes for each number of pixels to be changed can be stored and a draw (uniformly over the possibilities) can be made from this table. For one pixel change, only one possibility exist, for two pixel changes three possibilities exist, and for three pixel changes, ten possibilities exist. If a change of the pixels leads to an illegal configuration, no change of the contour is made (a change of the position node may however still be performed). In such cases, the diagonal elements in the $Q$-matrix will be non-zero. Note that for non-smooth contour, 

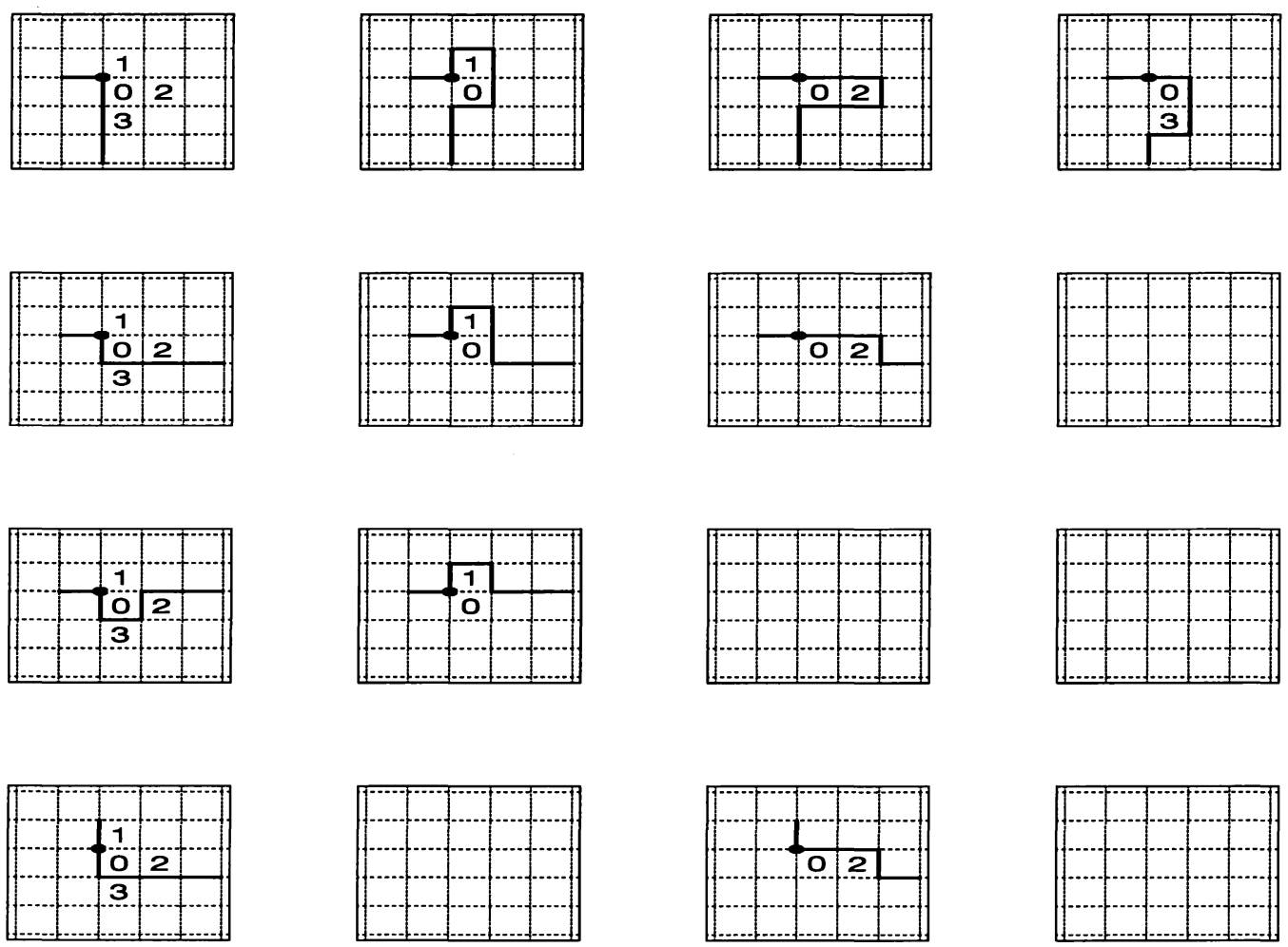

Figure 4: Examples of configurations and the possible changes when 2 pixels are assumed to be changed. The first column shows original contour, the first pixel to be changed (numbered 0) and the three possibilities for the second pixel to be changed (numbered 1,2,3). The second, third and fourth columns shows the new configurations for the three possibilities. If an illegal transition occurs, no contour is shown. The position node is marked with a dot.

many cases of illegal transitions occur. Furthermore, the chance of getting an illegal transition increases with the number of pixels to be changed.

For the $Q$-matrices described above and with uniform draws in each case, the algorithms simplify since $q_{x^{*} y^{*}}=q_{y^{*} x^{*}}$, implying that the Hastings extension of the Metropolis algorithm is not needed. The convergence rates of the algorithms largely depend on how smooth the contours are at each iteration step. Modifications of the draw for the positions of the pixels to be changed such that the contours are more likely to become smooth would presumably lead to faster convergence. In such cases, the Hastings extensions are necessary.

Consider now some alternative choices of the movement matrix $R$. For simulation, the only requirement on the movement matrix $R$ is that it is doubly stochastic, i.e. $\sum_{x} r_{x y}=1$, while irreducibility is required for optimization. We only consider choices of $R$ satisfying both these requirements.

Many different alternatives are possible. We consider a class where the distribution of the movement is independent of the position. To be more precise, let $m$ be the number of position movements made at a specific iteration step (that is, if $p^{x}=x_{p}$, a change of position node from $x_{p}$ to $x_{p+m}$ is performed, positive values correspond to movements clockwise, negative values to movements counter-clockwise). Assume $m$ is distributed according to some probability distribution $G$. It is easy to see that the independence of $m$ from $x$ implies that $R$ is doubly stochastic.

Any distribution $G$ is possible, but in practice, a distribution making a small number of 
movements should be preferred to minimize computation time. We will consider one particular case of $G$ where $m$ is uniformly distributed on the values $\left\{m_{l}, m_{l}+1, \ldots, m_{u}\right\}$. Still much freedom is available in the choice of $m_{l}$ and $m_{u}$. In Storvik [21], some simulation experiments were performed to compare the two choices $m_{l}=-3, m_{u}=3$ and $m_{l}=2, m_{u}=3$. The simulation experiments showed that the latter case performed better than the first. The reason may be that the algorithm performs best when changes are made smoothly along the contour corresponding to the choice of $m_{l}=2, m_{u}=3$.

Under the specifications described above, the algorithm 1 is given by the following.

Algorithm 3 Start with an arbitrary $X^{*}(0)$. For each iteration $s$ carry out the following steps:

1. Assume that $X^{*}(s)=x^{*}=\left(x, p^{x}\right)$ and select state $y^{*}=\left(y, p^{x}\right)$ by

(a) Draw the number of pixels to be changed.

(b) Decide if pixels on outside or inside of contour are to be changed (each with probability $0.5)$.

(c) Draw position of pixels to be changed.

(d) Decide if new configuration is a legal one.

2. If a legal configuration is obtained, change the contour to $z=y$ with probability $\alpha_{x^{*} y^{*}}$ while retain $z=x$ with probability $1-\alpha_{x^{*} y^{*}}$, where

$$
\alpha_{x^{*} y^{*}}=\frac{s_{x^{*} y^{*}}}{1+\frac{\pi^{*}\left(x^{*}\right)}{\pi^{*}\left(y^{*}\right)} \frac{q_{x^{*} *^{*}}}{q_{y^{*} x^{*}}}}
$$

and $S=\left\{s_{x^{*}}, s_{y^{*}}\right\}$ is a positive symmetric matrix.

If an illegal configuration is obtained, put $z=x$.

3. Draw the number of positions the position node is to be changed

4. Assuming $p^{x}=z_{p}$, put $p^{z}=z_{p+m}$.

5. Put $X^{*}(s+1)=\left(z, p^{z}\right)$.

\section{Applications}

In this section, we will present some results using the dynamic contour approach described in section 3. We consider two examples, one from ultrasound images of left ventricle, and one from Magnetic Resonance images of the human brain. Both examples will only be discussed briefly. For more thorough discussion of these problems, we refer to Storvik and Switzer [21], Storvik and Lundervold [20] and Lundervold and Storvik [15]. For both cases the implementation described in section 3.4 has been used. The optimization algorithm 2 with the annealing schedule $T(s)=c / \log (s+1)$ and with 1-3 pixels changed at each iteration step was used.

Consider first the ultrasound image of left ventricle. Figure 5 shows an example of such an image. The left ventricle is shown as the darker area in the middle of the image.

As a prior model for the contour, the fractal dimension (11) was used. The weight parameter corresponding to the prior energy function was 250.0. The likelihood of the observed image conditional on the contour was assumed to be the region-based Gaussian distribution (equations (14) and (15)). The parameters $\left(\mu_{1}, \sigma_{1}, \mu_{2}, \sigma_{2}\right)$ were estimated by training (drawing regions inside and outside the contour) on the observed image. The corresponding energy-function is given as minus the logarithm of the likelihood function (weight parameter equal to one). 


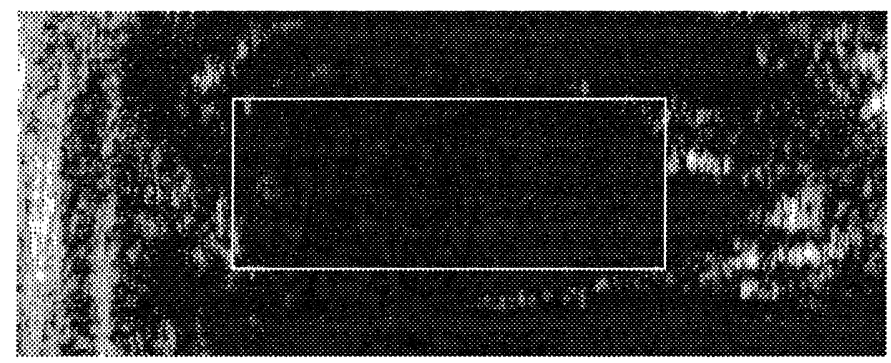

Figure 5: An ultrasound image of left ventricular cavity. The white curve indicate the initial configuration used for contour recognition.

In addition to the grey-level distributions specified above, the need for a gradient operator for recognizing edges in the vertical direction was recognized. In particular, an energy term was defined through potentials $V_{i}(x)$, where the potential function is given by

$$
\begin{array}{r}
V_{i}(x)=I\left(\operatorname{segment}\left(x_{i}, x_{i+1}\right) \text { horizontal }\right) \times \\
\qquad \begin{array}{ll}
-\left(z_{o}-z_{i}\right) & \text { if } z_{o}>z_{i} \\
-\frac{1}{1+z_{o}-z_{i}} & \text { otherwise }
\end{array}
\end{array}
$$

where $z_{i}$ and $z_{o}$ are the two pixels adjacent to the segment $\left(x_{i}, x_{i+1}\right)$ inside and outside the contour, respectively. Also this energy-term was given weight equal to one.

Figure 6 shows a restoration of the ventricle using algorithm 2 after 1000000 iterations (taking 137 seconds of computer time on a DECstation 5000/25) with $c=100$ (the initial contour is shown in figure 5). We see that the solution provides a very good fit to the contour of the left ventricle. Note that the contour is not particular smooth locally. This is because no local smoothness is incorporated into the prior.

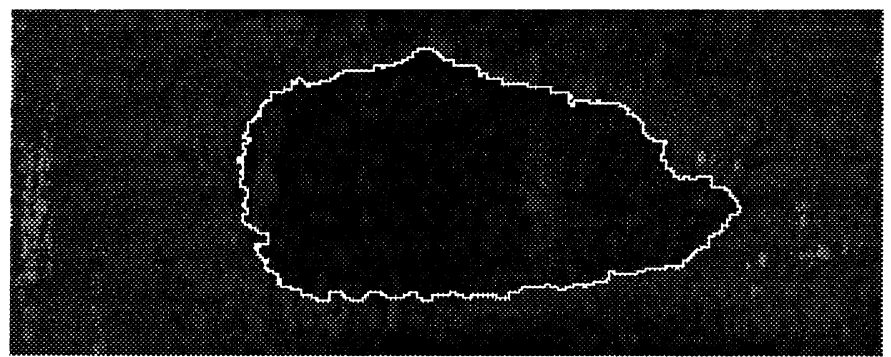

Figure 6: Restoration of the ultrasound image of left ventricular cavity.

The second example considered, is recognition of structures in an Magnetic Resonance (MR) image of the human brain. In this case, 4 channels are available, making the problem multispectral. An example is shown in Figure 7. In this case, three different contours are of interest. These contours are the boundary of the brain, the boundary of the ventricle system and the boundary between the brain parenchyma and the cerebro spinal fluid outside the brain parenchyma.

Each boundary were assumed to have independent priors (although no intersection between contours was allowed), specified through the energy corresponding to the fractal dimension (11), that is

$$
U_{1}(x)=\alpha_{1} U_{f}\left(x_{1}\right)+\alpha_{2} U_{f}\left(x_{2}\right)+\alpha_{3} U_{f}\left(x_{3}\right)
$$

where $U_{f}$ is given by equation (11) and $x_{1}, x_{2}$ and $x_{3}$ are the contours for the brain, the brain parenchyma and the ventricle system, respectively. The weight parameters $\alpha_{1}, \alpha_{2}$ and $\alpha_{3}$ were 


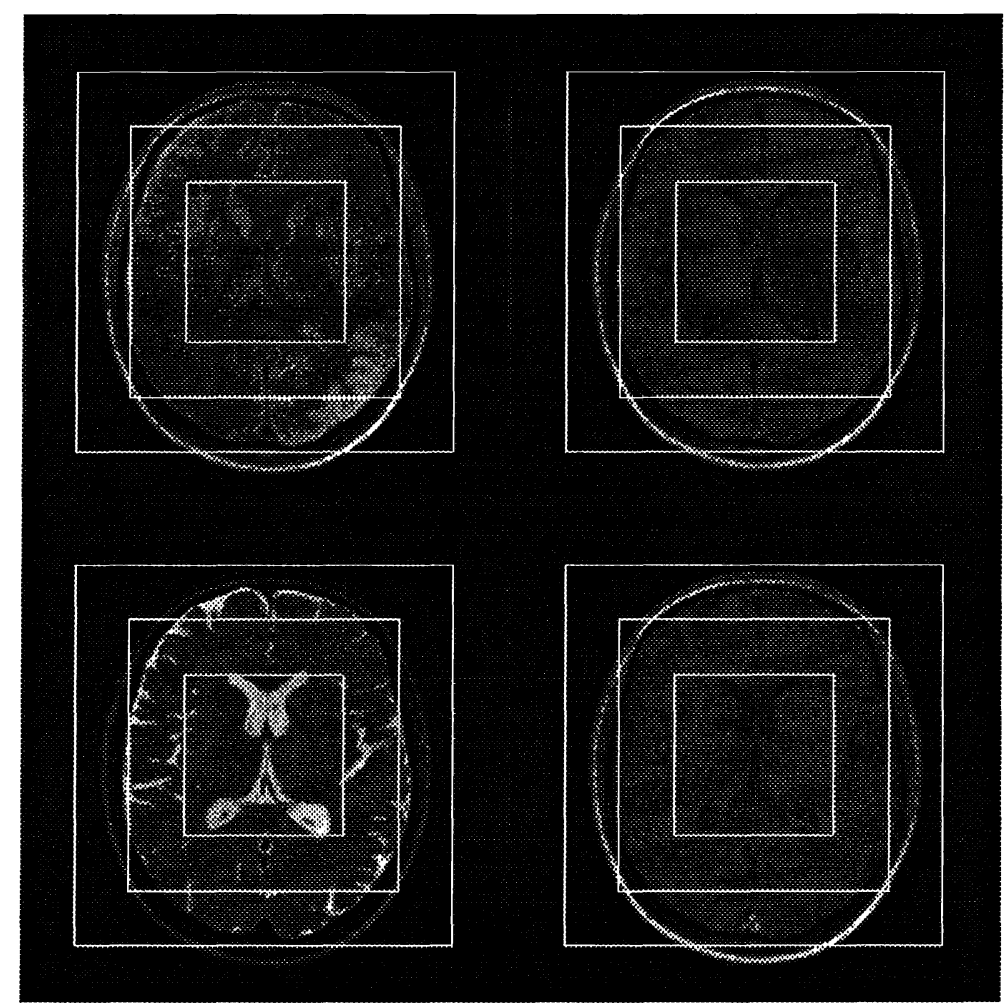

Figure 7: MR image from the human brain. Four channels, PD-weighted (upper left), T2weighted (upper right), T1-weighted (lower left) and T1-weighted with chemical contrast (lower right) are available. White curves indicate initial contours used for finding optimal contours.

chosen to be $1.0,0.3$ and 0.3 , indicating that the contour of the brain is more smooth than the other two contours.

The three contours divide the image into four regions. The pixels inside a region assumed to follow a mixture Gaussian distribution, that is

$$
z_{j} \sim \sum_{k=1}^{K_{i}} \pi_{i, k} N\left(\mu_{i, k}, \Sigma_{i, k}\right)
$$

for pixel $j$ in region $R_{i}$. The energy-function is given as minus the logarithm of the likelihood function (with weight parameter equal to one). The parameters $\left(\mu_{i, k}, \Sigma_{i, k}\right)$ were estimated by training from the same image. Storvik and Lundervold [20] and Lundervold and Storvik [15] considered an automatic procedure for estimation of these parameters.

Figure 8 shows a restoration of the MR images using algorithm 2 after 1000000 iterations (taking 548 seconds on a DECstation 5000/25) with $c=1$ (the initial contour is shown in figure 7). We see that also this solution gives a very good fit to the true contours. Note that although the images contain many edges, the method is able to distinguish between them with appropriate modeling of the likelihood function $f(z \mid x)$.

\section{Summary and discussion}

We have in this paper described a new method for identification contours of simply connected objects in (possible multispectral) images. The approach is based on the Bayesian paradigm, and is similar to the active contour approach in that the curve is moved dynamically to the solution, but differ in three important aspects: 1) more flexible models are possible to incorporate, 2) 


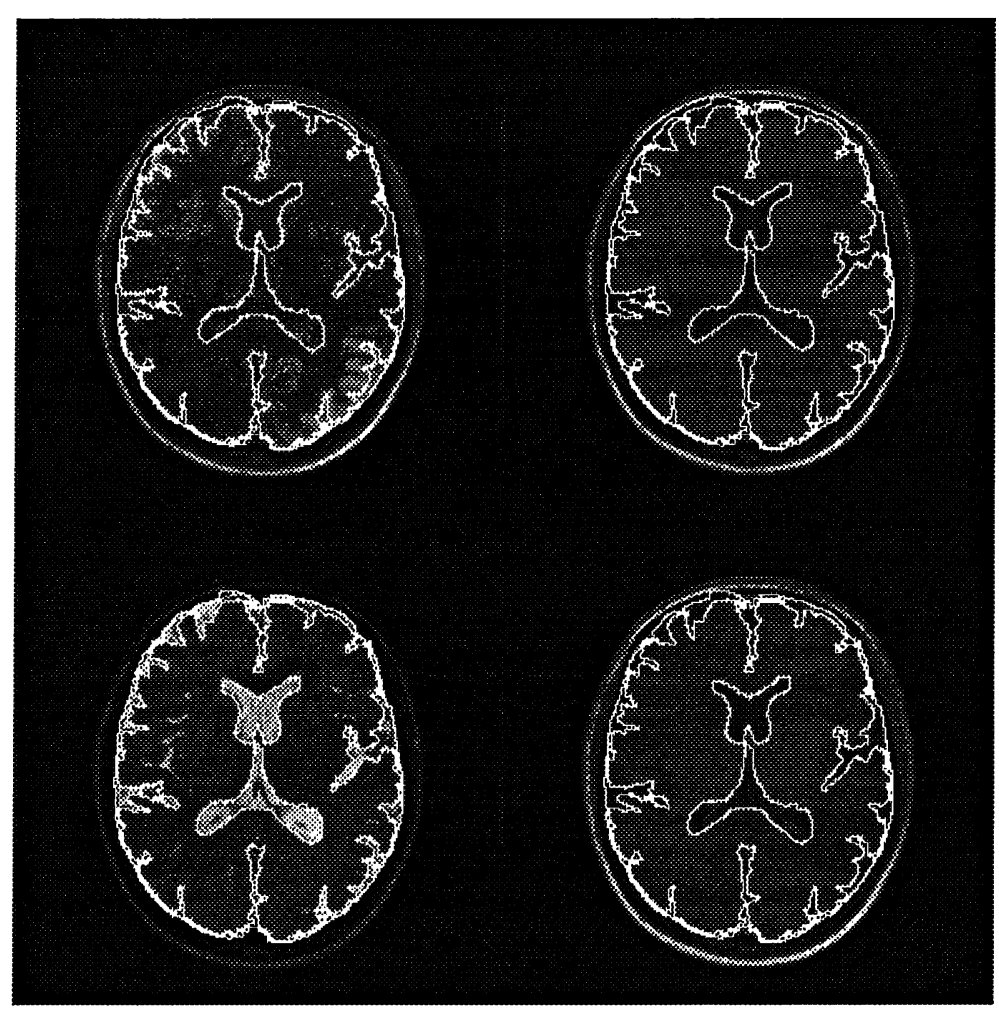

Figure 8: Restoration of contours in brain image.

algorithms that can find the global optimal solution independent of the initial configuration are used, and 3) algorithms to sample from the posterior distribution making estimates of uncertainty measures for any quantity of interest available.

Representation of contours and algorithms for simulation and optimization are discussed in a general framework. When actual implementation is to take place, specific choices has to be made. For the current implementation, a restriction on the representation is made to make the contours follow the pixel-sides. Such a restriction makes the likelihood functions for the observed images easy to calculate, but have some weaknesses concerning the actual contours. Improvements on the representation part is expected to give better results.

Improvements on the speed of convergence can be achieved by constructing algorithms allowing larger changes on the boundaries at each iteration step (in the current implementation only a few pixels (1-3) can be changed).

The method has been tried out on two real problems, ultrasound images of the left ventricle and Magnetic Resonance images of the human brain. In both cases promising results have been obtained. Parameters involved in the likelihood functions need to be estimated. The current approach has been to estimate by training (drawing regions in the observed image inside and outside the contours). Automatic methods for performing such estimation will be reported elsewhere.

This paper has been concentrating on contour detection in 2-dimensional images. Both the examples considered are actually 3-dimensional problems. The theoretical framework considered is relatively straightforward to extend to 3D. The increase of computational complexity is however extensive. Such an extension is under current investigation. 


\section{A Proof of Theorem}

Proof of Theorem 1: Since $Q$ and $R$ combine to an aperiodic and irreducible transition matrix, also $P$, the transition matrix of the Markov chain $\{X(s)\}$, is irreducible and aperiodic.

Note that without making further restrictions on $R$, in general $p_{x^{*} y^{*}}>0 \nRightarrow p_{y^{*} x^{*}}>0$. This makes it impossible to prove convergence by showing $\pi^{*}\left(x^{*}\right) p_{x^{*} y^{*}}=\pi^{*}\left(y^{*}\right) p_{y^{*} x^{*}}$ and the general results in Hastings [12] will not be valid. However, since the chain both is aperiodic and irreducible, it is sufficient to show that (see for example Ripley [19])

$$
\pi^{*}\left(y^{*}\right)=\sum_{x^{*}} \pi^{*}\left(x^{*}\right) p_{x^{*} y^{*}}
$$

for all $y^{*} \in \Omega^{*}$. Notice first that under the symmetry of positivity condition (25) and $p^{x}=p^{y}$,

$$
\begin{aligned}
\pi^{*}\left(x^{*}\right) & q_{x^{*} y^{*}} \alpha_{x^{*} y^{*}} \\
= & \pi^{*}\left(x^{*}\right) q_{x^{*} y^{*}} \frac{s_{x^{*} y^{*}}}{1+\frac{\pi^{*}\left(x^{*}\right)}{\pi^{*}\left(y^{*}\right)} \frac{q_{x^{*} y^{*}}}{q_{y^{*} x^{*}}}} \\
= & \pi^{*}\left(x^{*}\right) q_{x^{*} y^{*}} \frac{\pi^{*}\left(y^{*}\right)}{\pi^{*}\left(x^{*}\right)} \frac{q_{y^{*} x^{*}}}{q_{x^{*} y^{*}}} \frac{s_{y^{*} x^{*}}}{\frac{\pi^{*}\left(y^{*}\right)}{\pi^{*}\left(x^{*}\right)} \frac{q_{y^{*} x^{*}}}{q_{x^{*} y^{*}}}+1} \\
= & \pi^{*}\left(y^{*}\right) q_{y^{*} x^{*}} \alpha_{y^{*} x^{*}} .
\end{aligned}
$$

Further,

$$
\begin{aligned}
p_{x^{*}, y^{*}}= & q_{\left(x, p^{x}\right),\left(y, p^{x}\right)} \alpha_{\left(x, p^{x}\right)\left(y, p^{x}\right)} r_{\left(y, p^{x}\right)\left(y, p^{y}\right)}+ \\
& I(x=y) \sum_{z \in \Omega} q_{\left(y, p^{x}\right),\left(z, p^{x}\right)}\left(1-\alpha_{\left(y, p^{x}\right)\left(z, p^{x}\right)}\right) r_{\left(y, p^{x}\right)\left(y, p^{y}\right)}
\end{aligned}
$$

Let $p$ denote an arbitrary position node. Then

$$
\begin{aligned}
& \sum_{x^{*} \in \Omega^{*}} \pi^{*}\left(x^{*}\right) p_{x^{*} y^{*}} \\
& =\sum_{x \in \Omega} \sum_{p \in x} \pi^{*}(x, p) p_{(x, p)\left(y, p^{y}\right)} \\
& =\sum_{x \in \Omega} \sum_{p \in x} \pi^{*}(x, p)\left[q_{(x, p),(y, p)} \alpha_{(x, p)(y, p)} r_{(y, p)(y, p y)}+\right. \\
& \left.I(x=y) \sum_{z \in \Omega} q_{(y, p),(z, p)}\left(1-\alpha_{(y, p)(z, p)}\right) r_{(y, p)(y, p y)}\right] \\
& =\sum_{x \in \Omega} \sum_{p \in x} \pi^{*}(x, p) q_{(x, p)(y, p)} \alpha_{(x, p)(y, p)} r_{(y, p)\left(y, p^{y}\right)} \\
& +\sum_{p \in y} \sum_{z \in \Omega} \pi^{*}(y, p) q_{(y, p)(z, p)}\left(1-\alpha_{(y, p)(z, p)}\right) r_{(y, p)(y, p y)} \\
& \stackrel{(35)}{=} \sum_{x \in \Omega} \sum_{p \in x} \pi^{*}(y, p) q_{(y, p)(x, p)} \alpha_{(y, p)(x, p)} r_{(y, p)(y, p y)} \\
& +\sum_{p \in y} \sum_{z \in \Omega} \pi^{*}(y, p) q_{(y, p)(z, p)}\left(1-\alpha_{(y, p)(z, p)}\right) r_{(y, p)(y, p y)} \\
& \stackrel{(*)}{=} \pi^{*}(y, p) \sum_{x \in \Omega} \sum_{p \in x} q_{(y, p)(x, p)} \alpha_{(y, p)(x, p)} r_{(y, p)\left(y, p^{y}\right)} \\
& +\pi^{*}(y, p) \sum_{p \in y} \sum_{x \in \Omega} q_{(y, p)(x, p)}\left(1-\alpha_{(y, p)(x, p)}\right) r_{(y, p)\left(y, p^{y}\right)}
\end{aligned}
$$

where in $(*)$ it is used that $\pi^{*}(y, p)$ is uniform in $p$ (model $\left.(20)\right)$. Now, 


$$
q_{(y, p)(x, p)}>0 \Rightarrow p \in x \text { and } p \in y
$$

giving

$$
\begin{aligned}
\sum_{x \in \Omega} \sum_{p \in x} q_{(y, p)(x, p)} & \alpha_{(y, p)(x, p)} r_{(y, p)\left(y, p^{y}\right)} \\
= & \sum_{x \in \Omega} \sum_{p \in y} q_{(y, p)(x, p)} \alpha_{(y, p)(x, p)} r_{(y, p)\left(y, p^{y}\right)} \\
& =\sum_{p \in y} \sum_{x \in \Omega} q_{(y, p)(x, p)} \alpha_{(y, p)(x, p)} r_{(y, p)\left(y, p^{y}\right)}
\end{aligned}
$$

Using this, we get

$$
\begin{aligned}
\sum_{x^{*} \in \Omega^{*}} & \pi^{*}\left(x^{*}\right) p_{x^{*} y^{*}} \\
= & \pi^{*}(y, p) \sum_{p \in y} \sum_{x \in \Omega} q_{(y, p)(x, p)} \alpha_{(y, p)(x, p)} r_{(y, p)\left(y, p^{y}\right)}+ \\
& \pi^{*}(y, p) \sum_{p \in y} \sum_{x \in \Omega} q_{(y, p)(x, p)}\left(1-\alpha_{(y, p)(x, p)}\right) r_{(y, p)(y, p y)} \\
= & \pi^{*}\left(y^{*}\right) \sum_{p \in y} \sum_{x \in \Omega} q_{(y, p)(x, p)} r_{(y, p)(y, p y)} \\
= & \pi^{*}\left(y^{*}\right) \sum_{p \in y} r_{(y, p)(y, p y)} \sum_{x \in \Omega} q_{(y, p)(x, p)} \\
= & \pi^{*}\left(y^{*}\right) \sum_{p \in y} r_{(y, p)(y, p y)} \\
\stackrel{(26)}{=} & \pi^{*}\left(y^{*}\right)
\end{aligned}
$$

This concludes the proof.

Proof of Theorem 2: The convergence proof in this case is based on the results in Hajek [11]. We will state this result, after introducing some notation and definitions (also taken from Hajek [11]):

For each state $x$ in $\Omega$, define $\mathcal{N}(x) \subset \Omega$, called the neighbors of $x$. Typically the sets $\mathcal{N}(x)$ are small subsets of $\Omega$.

Definition 1 (Reachability) State $y$ is reachable at height $E$ from state $x$ if $x=y$ and $U(x) \leq E$, or if there is a sequence of states $x^{0}=x, x^{1}, \ldots, x^{p}=y$ for some $p \geq 1$ such that $x^{k+1} \in \mathcal{N}\left(x^{k}\right)$ for $0 \leq k<p$ and $U\left(x^{k}\right) \leq E$ for $0 \leq k \leq p$.

Property 1 (Weak reversibility (WR)) For any real number $E$ and any two states $x$ and $y, x$ is reachable at height $E$ from $y$ if and only if $y$ is reachable at height $E$ from $x$.

Definition 2 (Local minimum) State $x$ is said to be a local minimum if no state $y$ with $U(y)<U(x)$ is reachable from $x$ at height $U(x)$. The depth of a local minimum $x$ is defined to be plus infinity if $x$ is a global minimum. Otherwise, the depth of $x$ is the smallest number $E, E>0$, such that some state $y$ with $U(y)<U(x)$ can be reached from $x$ at height $U(x)+E$.

We can then present (in Hajek [11] a more general version for continuous Markov processes were given, but we will need only the results for discrete time Markov chains): 
Theorem 3 (Hajek) Let $X(s)$ be a Markov chain with state space $\Omega$, set of neighbors $\mathcal{N}(x)$ and one-step transition transition-probabilities $\operatorname{Pr}[X(s+1)=y \mid X(s)=x]=P\left(x, y, s, \lambda_{s}\right)$. Let further $U(x)$ be defined over $\Omega$. Assume that the following holds:

1. $(\Omega, P, \mathcal{N})$ is irreducible.

2. The weak reversibility property holds.

3. For all $s \geq 0$ there exists some strictly positive constants $c_{1}, c_{2}$ and $0 \leq \lambda_{s} \leq 1$ such that $P\left(\cdot, \cdot, s, \lambda_{s}\right)$ is a probability transition matrix satisfying

$$
c_{1} \lambda_{s}^{(U(y)-U(x))^{+}} \leq P\left(x, y, s, \lambda_{s}\right) \leq c_{2} \lambda_{s}^{(U(y)-U(x))^{+}}
$$

if $y \in \mathcal{N}(x)$, and

$$
P\left(x, y, s, \lambda_{s}\right)=0
$$

if $y \notin \mathcal{N}(x) \bigcup\{x\}$.

4. $\left\{\lambda_{s}\right\}$ is non-increasing in $s$ and $\lim _{s \rightarrow \infty}=0$.

Then

(a) For any state $x$ that is not a local minimum of $U(x), \lim _{s \rightarrow \infty} \operatorname{Pr}[X(s)=x]=0$.

(b) Let $d^{*}$ be the maximum of the depths of all states which are local but not global minima. Let $\Omega^{M}$ denote the set of global minima of $U(x)$. Then $\lim _{s \rightarrow \infty} \operatorname{Pr}[X(s) \in$ $\left.\Omega^{M}\right]=1$ if and only if $\sum_{0}^{\infty} \lambda_{s}^{d^{*}}=+\infty$.

The parameter $\lambda_{s}$ is closely related to $T(s)$ and typically $\lambda_{s}=e^{-1 / T(s)}$.

We are then ready to prove Theorem 2 by applying Theorem 3 . Note that $\Omega, x$ and $y$ in Theorem 3 correspond to $\Omega^{*}, x^{*}$ and $y^{*}$ in our notation. The first condition in Theorem 3 is satisfied by definition. For each $x^{*}=\left(x, p^{x}\right) \in \Omega$, define $\mathcal{N}\left(x^{*}\right)$ by

$$
\begin{aligned}
\mathcal{N}\left(x^{*}\right)= & \left\{y^{*} ; q_{\left(x, p^{x}\right)\left(y, p^{x}\right)}>0 \text { and } r_{\left(y, p^{x}\right)\left(y, p^{y}\right)>0}\right\} \\
& \left\{y^{*} ; y=x \text { and } r_{\left(x, p^{x}\right)\left(x, p^{y}\right)>0}\right\}
\end{aligned}
$$

In this case $y^{*} \in \mathcal{N}\left(x^{*}\right)$ will not in general imply that $x^{*} \in \mathcal{N}\left(y^{*}\right)$. To prove that the WR property holds, we therefore have to show this in another way.

Assume that $x^{*}$ is reachable at height $E$ from $y^{*}$. Let $\left\{x_{0}^{*}, \ldots, x_{p}^{*}\right\}$ be the sequence of states implying the reachability condition. Assuming $x_{k}^{*}$ is reachable at height $E$ from $x_{k+1}^{*}$, it follows that $y^{*}$ is reachable at height $E$ from $x^{*}$. It therefore suffices to show that $x_{k}^{*}$ is reachable at height $E$ from $x_{k+1}^{*}$.

If $x_{k}=x_{k+1}$, then by the condition on irreducibility of $R$, there exists a sequence $x_{k+1}^{*}=$ $z_{0}^{*}, z_{1}^{*}, \ldots, z_{q}^{*}=x_{k}^{*}$ such that $z_{j}^{*}=x_{k}^{*}$ for $0 \leq j \leq q$ and $z_{j+1}^{*} \in \mathcal{N}\left(z_{j}^{*}\right)$.

If $x_{k} \neq x_{k+1}$, then $p^{x_{k}}$ is a node on $x_{k+1}$ because of the restriction that the position node is not to be removed under a change of the contour. Again by the condition on irreducibility of $R$, there exists a sequence $x_{k+1}^{*}=z_{0}^{*}, z_{1}^{*}, \ldots, z_{q}^{*}=\left(x_{k+1}, p^{x_{k}}\right)$ such that $z_{j}=x_{k+1}$ and $z_{j+1}^{*} \in \mathcal{N}\left(z_{j}^{*}\right)$. Furthermore, since $q_{\left(x_{k}, p^{x_{k}}\right)\left(x_{k+1}, p^{x_{k}}\right)}>0$, by the symmetry in positivity condition also $q_{\left(x_{k+1}, p^{x_{k}}\right)\left(x_{k}, p^{x_{k}}\right)}>0$ and since both $U(x) \leq E$ and $U(y) \leq E, x_{k}^{*}$ is reachable at height $E$ from $x_{k+1}^{*}$. This proves the weak reversibility condition.

The transition matrix is in this case

$$
\begin{aligned}
p_{x^{*} y^{*}} & =q_{\left(x, p^{x}\right)\left(y, p^{x}\right)} \alpha_{x^{*}, y^{*}} r_{\left(y, p^{x}\right)\left(y, p^{y}\right)} \\
& =q_{\left(x, p^{x}\right)\left(y, p^{x}\right)} e^{-(U(y)-U(x))^{+} / T(s)} r_{\left(y, p^{x}\right)\left(y, p^{y}\right)}
\end{aligned}
$$


for $x \neq y$, and

$$
\begin{aligned}
p_{x^{*} y^{*}} & =\sum_{z=\left(z, p^{x}\right)} q_{x^{*} z^{*}}\left(1-\alpha_{x^{*}, y^{*}}\right) r_{\left(x, p^{x}\right)\left(x, p^{y}\right)} \\
& =\sum_{z=\left(z, p^{x}\right)} q_{x^{*} z^{*}}\left(1-e^{-(U(z)-U(x))^{+} / T(s)}\right) r_{\left(x, p^{x}\right)\left(x, p^{y}\right)}
\end{aligned}
$$

for $x=y$. Choosing $\lambda_{s}=e^{-1 / T(s)}, c_{1}=\min \left\{p_{x^{*} y^{*}} ; p_{x^{*} y^{*}}>0\right\}$ and $c_{2}=\max p_{x^{*} y^{*}}$, fulfills the third condition in Theorem 3 , concluding the proof.

\section{Acknowledgment}

I wish to thank my supervisor professor Erik Bølviken for valuable guidance and discussions throughout this work. I further thank the referees for valuable comments on the paper.

\section{References}

[1] A. A. Amini, S. Tehrani, and T. E. Weymouth. Using dynamic programming for minimizing the energy of active contours in the presence of hard constraints. In Proc. Second Int. Conf. Computer Vision, pages 95-99, Tarpon Springs, FL, December 1988.

[2] A. A. Amini, T. E. Weymouth, and R. C. Jain. Using dynamic programming for solving variational problems in vision. IEEE Transactions on Pattern Analysis and Machine Intelligence, 12:855-867, 1990.

[3] J. Besag. Towards Bayesian image analysis. Journal of Applied statistics, 16(3):395-407, 1989.

[4] O. Cantoni. Rough large deviation estimates for simulated annealing: Application to exponential schedules. Ann. Probab., 20(3):1109-1146, 1992.

[5] I. Cohen, L. D. Cohen, and N. Ayache. Using Deformable Surfaces to Segment 3-D Images and Infer Differential Structures. CVGIP: Image understanding, 56(2):242-263, 1992.

[6] L. D. Cohen. On active contour models and balloons. CVGIP: Image understanding, 52(2):211-218, 1991.

[7] T. E. Dufresne and A. P. Dhawan. A Structured Approach to Edge Clustering and Extrapolation. In IEEE Int. Conf. on Systems Engineering, pages 262-65, Fairborn, Ohio, August 1991.

[8] N. Friedland and D. Adam. Ventricular cavity boundary detection from sequential ultrasound images using simulated annealing. IEEE Transactions on Medical Imaging, 8(4), 1989.

[9] A. E. Gelfand and A. F. M. Smith. Sampling-based approaches to calculating marginal densities. Journal of the American Statistical Association, 85(410):398-409, 1990.

[10] S. Geman and D. Geman. Stochastic relaxation, Gibbs distribution, and Bayesian restoration of images. IEEE Transactions on Pattern Analysis and Machine Intelligence, 6(6):721741,1984

[11] B. Hajek. Cooling schedules for optimal annealing. Mathematics of operation research, 13(2):311-329, 1988. 
[12] W. K. Hastings. Monte Carlo sampling methods using Markov chains and their applications. Biometrika, 57(1):97-109, 1970.

[13] M. Kass, A. Witkin, and D. Terzopoulos. Snakes: Active contour models. Int. J. Comput. Vision, 1(4):321-331, 1988.

[14] F. Leitner, I. Marque, S. Lavallée, and P. Cinquin. Dynamic Segmentation: Finding the Edge With Snake Splines. In P. J. Laurent, A. Le Méhauté, and L. Schumaker, editors, Curves and Surfaces, pages 279-284. Academic Press, Boston, 1991.

[15] A. Lundervold and G. Storvik. Segmentation of Brain Parenchyma and Cerebrospinal Fluid in Multispectral Magnetic Resonance Images. Manuscript under preparation, 1993.

[16] A. Lundervold and T. Taxt. Automatic detection of left ventricular cardiac boundary. In NOBIM Conference, Troms $\varnothing$, May 1990.

[17] N. Metropolis, A. W. Rosenbluth, M. N. Rosenbluth, A. H. Teller, and E. Teller. Equations of state calculations by fast computing machines. J. Chem. Phys., 21:1087-1092, 1953.

[18] E. Persoon and K. S. Fu. Shape discrimination using fourier descriptors. IEEE Transactions on Systems, Man, Cybernetics, 7:171-179, 1977.

[19] B. D. Ripley. Stochastic simulation. John Wiley \& Sons, New York, 1987.

[20] G. Storvik and A. Lundervold. Segmentation of Brain Parenchyma and CSF in Multispectral MR Images of the Head. The 8th SCIA Conference, May 1993.

[21] G. Storvik and P. Switzer. Space-time modeling of simply connected objects: an application to detection of left ventricular cardiac boundaries from ultrasound images. In Interface'92, Texas, March 1992.

[22] H. L. Tan, S. B. Gelfand, and E. J. Delp. A Cost Minimisation Approach to Edge Detection Using Simulated Annealing. IEEE Transactions on Pattern Analysis and Machine Intelligence, 14(1):3-17, 1991.

[23] V. Venkateswar and R. Chellappa. Extraction of Straight Lines in Aerial Images. IEEE Transactions on Pattern Analysis and Machine Intelligence, 14(11):1111-1114, 1992.

[24] D. J. Williams and M. Shah. A fast algorithm for active contours and curvature estimation. CVGIP: Image understanding, 55(1):14-26, 1992.

[25] C. T. Zahn and R. Z. Roskies. Fourier descriptors for plane closed contour. IEEE Transactions on Computers, 21:269-281, 1972. 